\title{
Dominant effect of ethanol in thermal destabilization of bovine serum albumin in the presence of sucrose
}

\author{
V. Sathya Devi ${ }^{*}$, Obiora O. Chidi ${ }^{* *}$ and Denis Coleman \\ Solus Biosystems, Palo Alto, CA, USA
}

\begin{abstract}
The thermal melting of bovine serum albumin (BSA) in the presence of excipients like ethanol and sucrose was studied by circular dichroism spectroscopy at physiological $\mathrm{pH}$ in phosphate buffered saline. Calculated apparent $T_{m}$ values were used to assess the thermal stability using two state fitted experimental curves. $0.5 \mathrm{M}$ sucrose stabilized the BSA indicated by the increase in $T_{m}$ of $\sim 8^{\circ} \mathrm{C}$ when compared to the $T_{m}$ of the same solution measured in the absence of sucrose. Conversely, in the presence of varying concentrations of ethanol (2-20\%), the protein was destabilized by a decrease of $\sim 3-10^{\circ} \mathrm{C}$ of $T_{m}$. In the binary mixture of sucrose and ethanol, the $T_{m}$ values showed that ethanol dominantly destabilized BSA in the presence of sucrose, possibly by weakening the hydrophobic interactions in the protein.
\end{abstract}

Keywords: Ethanol, sucrose, bovine serum albumin, circular dichroism spectroscopy

\section{Introduction}

Alcohols such as ethanol critically affect many biological processes which includes neurotransmission and immune responses [6,9]. Studying the effect of ethanol on the conformational stability of proteins like albumin is essential since albumin is the most abundant blood plasma protein that plays key role in the transport of hormones, anesthetics, endogenous ligands and fatty acids in blood [5]. Bovine serum albumin (BSA), which is homologous to human serum albumin (HSA), is a single polypeptide chain with 583 amino acid residues and three domains named I-III. Each domain is subdivided into two more domains (A and B) that are predominantly helical and cross-linked by several disulfide bridges [4]. Direct binding of ethanol to the hydrophobic binding sites on fatty acid free bovine serum albumin (BSA) was reported [1]. The study showed that the presence of ethanol inhibited the binding of 1-anilinonaphthalene-8-sulfonic acid (1, 8-ANS) and cis-parinaric acid to BSA. Also, ethanol's effect on the thermal stability of HSA was studied by Differential scanning calorimetry (DSC) very recently [13]. The study showed that the presence of ethanol decreased the thermal stability of HSA. The effect of ethanol in decreasing the thermal stability of proteins is attributed to the perturbation of the intramolecular hydrophobic interactions [8].

While ethanol thermally destabilizes these proteins, sugars such as sucrose are generally known to stabilize proteins. Sucrose stabilization is explained by the preferential exclusion phenomenon [12].

\footnotetext{
${ }^{*}$ Corresponding author: V. Sathya Devi, Solus Biosystems Inc., 2454 Embarcadero Way, Palo Alto, CA 94305, USA. Tel.: +1 650521 7703; Fax: +1 650251 9832; E-mail: sathya@ solusbiosystems.com.

${ }^{* *}$ Present address: Boston University School of Public Health, 715 Albany Street, Boston, MA, USA.
} 
Proteins are preferentially hydrated in the presence of sucrose which enhances the thermal stability of the same [2]. Understanding protein stabilization and destabilization mechanisms in a multi solvent system is garnering attention specifically in industries due to formulation efforts [16]. The effects of stabilizing and destabilizing cosolvents on proteins have been studied separately but their combined effects on altering protein's physicochemical properties are limited. Here we have attempted to study the combined effect of ethanol and sucrose on the thermal stability of BSA by circular dichroism spectroscopy.

\section{Materials and methods}

\subsection{Materials}

Bovine serum albumin (globulin free of $96 \%$ purity) was purchased from Sigma Aldrich. The protein was dialyzed overnight against $20 \mathrm{mM}$ sodium phosphate buffer, $\mathrm{pH} 7.0$. The concentration of BSA $(0.2 \mathrm{mg} / \mathrm{ml})$ was measured by UV Spectrophotometer using E1\% $1 \mathrm{~cm}=6.8$ at $280 \mathrm{~nm} \mathrm{[10].} \mathrm{The}$ sucrose concentration was $0.5 \mathrm{M}$ while the ethanol concentrations varied between $2 \%$ and $20 \% \mathrm{v} / \mathrm{v}$. BSA samples were incubated with ethanol and sucrose for 30 minutes before the measurements. The $\mathrm{pH}$ of the albumin solutions was not affected by ethanol and sucrose.

\subsection{Circular dichroism spectroscopy}

CD measurements were taken with a Jasco 715 Spectropolarimeter equipped with a computercontrolled water bath, and samples were placed in a cylindrical jacketed cuvette of $1 \mathrm{~mm}$ optical path length. The thermal melting was monitored at $222 \mathrm{~nm}$ from $25^{\circ} \mathrm{C}$ to $95^{\circ} \mathrm{C}$ at $1^{\circ} \mathrm{C} / \mathrm{min}$ rate. The thermal unfolding of BSA is only partially reversible when cooled back. Therefore the $T_{m}$ values that were calculated in this study by fitting with two state equations [15] are apparent $T_{m}$ values. Also, the stability that is mentioned throughout this article is related to the $\alpha$-helical structure of BSA resisting thermal denaturation.

\section{Results and discussion}

\subsection{Effect of 0.5 M sucrose on the thermal stability of BSA}

The CD thermal melting curves for both BSA and BSA in the presence of $0.5 \mathrm{M}$ sucrose $\left(\mathrm{BSA}_{\mathrm{suc}}\right)$ are shown in Fig. 1. To facilitate the comparison, the mdeg values were normalized for BSA concentration. The starting ellipticity value is consistent with the literature [11]. Both samples were heated from $25^{\circ} \mathrm{C}$ to $95^{\circ} \mathrm{C}$ at $1^{\circ} \mathrm{C} / \mathrm{min}$ rate, and cooled back to check for the protein's reversibility. The cooling curves were only partially reversible (data not shown). The apparent $T_{m}$ values that were calculated using two state fitting equations are shown in Table 1. BSA does not lose its secondary structure even when heated to $95^{\circ} \mathrm{C}$ probably due to the constraint of disulfide bonds. The presence of sucrose stabilizes BSA by about $8^{\circ} \mathrm{C}$. One similar study where BSA was heated in the presence of $40 \%$ sucrose by DSC showed an increase of $7^{\circ} \mathrm{C}$ [2]. Sucrose does not induce conformational changes at lower temperatures, as evident by the overlay of the melting curves of both samples up to $55^{\circ} \mathrm{C}$, which is the onset temperature of 


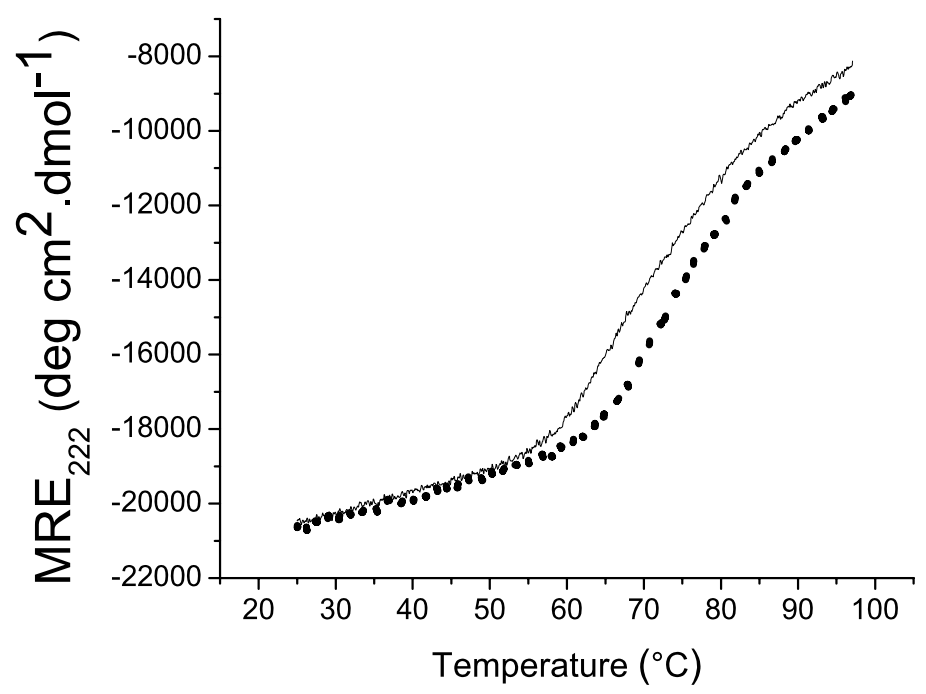

Fig. 1. Thermal melting curves of BSA monitored at $222 \mathrm{~nm}$ by CD spectroscopy. The change of mean residue ellipticity at $222 \mathrm{~nm}$ with temperature upon continuous heating $\left(1^{\circ} \mathrm{C} / \mathrm{min}\right)$ is shown both in the absence (solid) and presence (dot) of $0.5 \mathrm{M}$ sucrose. The apparent $T_{m}$ values calculated from the two state fitting of experimental curves are shown in Table 1 . Presence of sucrose increased both the onset and melting temperatures of BSA.

Table 1

\begin{tabular}{lc} 
Apparent $T_{m}$ values calculated for BSA and \\
BSA suc in varying concentrations of ethanol $^{\text {Sample }}$ & Apparent $T_{m}\left({ }^{\circ} \mathrm{C}\right)$ \\
\hline BSA & $70.2 \pm 0.1$ \\
In ethanol & \\
$2 \%$ & $67.9 \pm 0.07$ \\
$5 \%$ & $66.7 \pm 0.06$ \\
$10 \%$ & $67.0 \pm 0.07$ \\
$20 \%$ & $59.9 \pm 0.05$ \\
BSA $_{\text {suc }}$ & $78.1 \pm 0.3$ \\
In ethanol & \\
$2 \%$ & $70.5 \pm 0.04$ \\
$5 \%$ & $69.4 \pm 0.12$ \\
$10 \%$ & $69.4 \pm 0.10$ \\
$20 \%$ & $60.0 \pm 0.05$ \\
\hline
\end{tabular}

unfolding for BSA. The onset temperature of $\mathrm{BSA}_{\text {suc }}$ is higher by $5-7^{\circ} \mathrm{C}$. Sucrose is preferentially depleted from proteins surface which in turn strengthens the water structure around the protein molecules and hence intensifies the hydrophobic interactions in the protein [3]. This explains the enhanced thermal stability of BSA $_{\text {suc }}$.

\subsection{Effect of ethanol on the thermal stability of BSA}

Figure 2 shows the effect of increasing the concentration of ethanol on the thermal stability of BSA. While the apparent $T_{m}$ value of BSA is $70.2^{\circ} \mathrm{C}$, the presence of ethanol destabilizes BSA thereby de- 


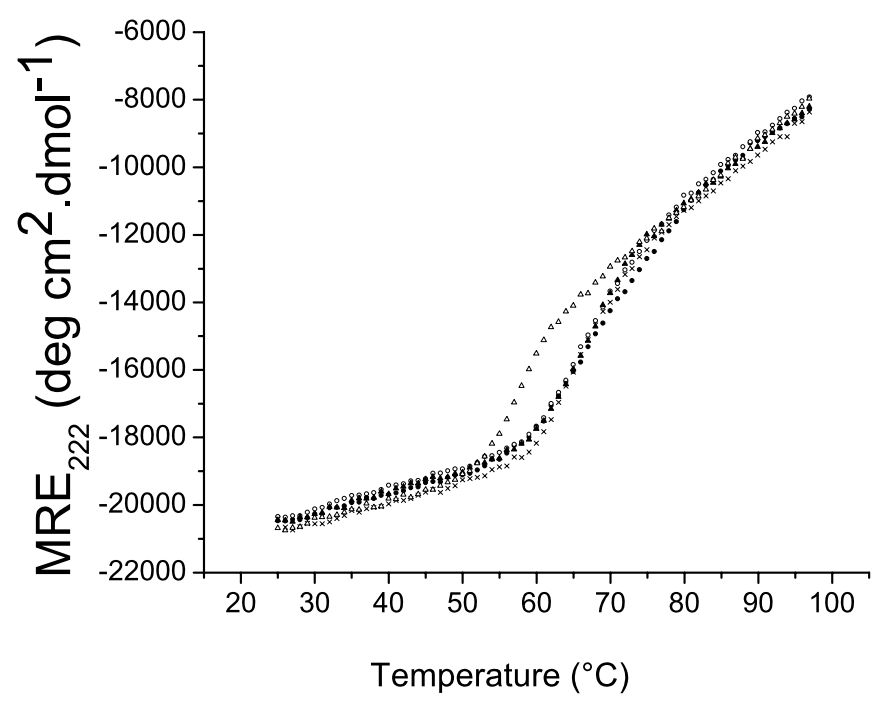

Fig. 2. Thermal melting curves of BSA monitored at $222 \mathrm{~nm}$ by CD spectroscopy in the presence of $0 \%$ (filled circle), $2 \%$ (open circle), $5 \%$ (cross), $10 \%$ (filled triangle) and $20 \%$ (open triangle) of ethanol. The apparent $T_{m}$ values (Table 1) calculated from the fitting of the experimental curves show that as the concentration of ethanol increases, the thermal stability of BSA decreases.

creasing the $T_{m}$ values (Table 1). An ethanol concentration less than $1 \% \mathrm{v} / \mathrm{v}$ does not have an effect on the thermal stability of BSA. Thus the data shown here are from $2 \% \mathrm{v} / \mathrm{v}$ concentration, which decreased the $T_{m}$ by $3^{\circ} \mathrm{C}$. The thermal stability is not affected between $5 \%$ and $10 \% \mathrm{v} / \mathrm{v}$ ethanol concentration. $T_{m}$ values remain constant between those concentrations of ethanol, but $20 \% \mathrm{v} / \mathrm{v}$ ethanol decreases the $T_{m}$ by almost $10^{\circ} \mathrm{C}$. Direct binding of ethanol to BSA was studied by Fluorescent and ${ }^{13} \mathrm{C}$ NMR [1]. In this study, it was found that ethanol prevents the binding of cis-parinaric acid to three out of five fatty acid binding sites in BSA. It was concluded that ethanol is selective in its interaction with the hydrophobic sites of BSA because the methyl group of ethanol was discovered to bind to the hydrophobic pocket of BSA. Our study found that binding of ethanol decreases the thermal stability of BSA which can be explained as follows. Ethanol competes with the bound endogenous fatty acids in BSA and most likely replaces them. This substitution disrupts the intramolecular hydrophobic interactions. Disruption of hydrophobic interactions naturally leads to a decrease in the thermal stability. We have not observed any induction of non-native helical structures of BSA in the presence of ethanol. Because it is highly hydrophobic with a lower dielectric constant, it might not have a strong influence in inducing non native secondary structure except for protein denaturation [14].

\subsection{Effect of ethanol on $B S A_{\text {suc }}$}

In order to understand the combined effects of sucrose and ethanol, we studied the influence of varying concentrations of ethanol on the thermal stability of $\mathrm{BSA}_{\text {suc }}$ (Fig. 3). The dominant effect of ethanol in destabilizing $\mathrm{BSA}_{\text {suc }}$ is clearly shown by the apparent $T_{m}$ values (Table 1). Despite of being stabilized by $0.5 \mathrm{M}$ sucrose, the presence of even $2 \% \mathrm{v} / \mathrm{v}$ ethanol decreases the $T_{m}$ by almost $8^{\circ} \mathrm{C}$. As observed in Fig. 2, the $T_{m}$ values remain constant between $5 \%$ and $10 \% \mathrm{v} / \mathrm{v}$ ethanol and decreased further by $20^{\circ} \mathrm{C}$ in $20 \% \mathrm{v} / \mathrm{v}$ ethanol. Stabilizing solvents maintain or increase the hydration of protein while destabilizing solvents directly bind to the protein and dehydrate them. Ethanol directly binds to the proteins disrupting the hydrophobic interactions while sucrose is preferentially depleted from proteins surface enhancing 


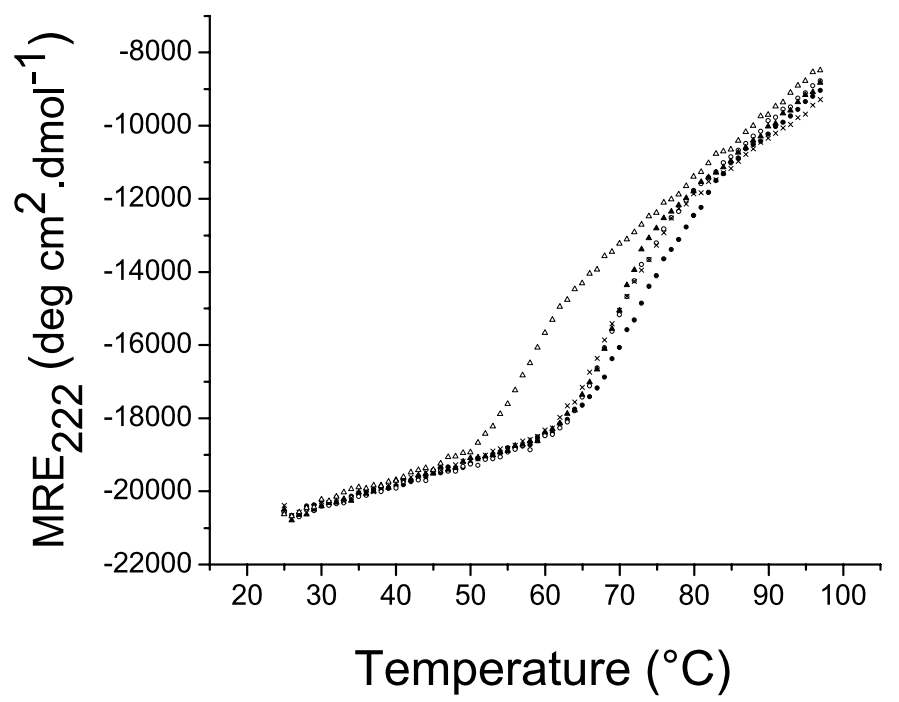

Fig. 3. Thermal melting curves of $\mathrm{BSA}_{\text {suc }}$ monitored at $222 \mathrm{~nm}$ by CD spectroscopy in the presence of $0 \%$ (filled circle), $2 \%$ (open circle), $5 \%$ (cross), 10\% (filled triangle) and 20\% (open triangle) of ethanol. Ethanol dominantly decreased the thermal stability of BSA which was otherwise stabilized by sucrose.

the hydration and hence the stability. However from our study, when considering the combined effect, it looks like effect of ethanol dominates the effect of sucrose on the stability of BSA. Solvent interactions provide complex contributions to protein folding through hydration, hydrogen bonding, hydrophobic interaction, van der Waals forces and electrostatic interactions. Out of which, hydrophobic interaction is thought to be the dominant force in governing the stability of proteins [7]. Our study made the same observation. In the presence of sucrose, hydrophobic interactions are enhanced due to hydration effects. On the other hand, the addition of ethanol disrupts the hydrophobic interactions, thermally destabilizing the protein more than in the absence of sucrose. Ethanol is much stronger in destabilizing BSA even in the presence of a stabilizer.

\section{Conclusions}

Serum albumins are excellent targets not only for biochemical investigations but also for pharmaceutical industries to characterize the effect of different solvents on their stability as they are used as therapeutic agents. Here we have used BSA to study the combined effect of ethanol and sucrose. Our $\mathrm{CD}$ thermal melting experiments highlight the dominant influence of ethanol in destabilizing $\mathrm{BSA}_{\text {suc }}$ by disrupting the hydrophobic interactions. These kinds of studies are important to understanding the role played by the combined use of binary cosolvents in protein stabilization.

\section{References}

[1] N.A. Avdulov, S.V. Chochina, V.A. Daragan, F. Schroeder, K.H. Mayo and W.G. Wood, Direct binding of ethanol to bovine serum albumin: a fluorescent and ${ }^{13} \mathrm{C}$ NMR multiplet relaxation study, Biochemistry 35 (1996), 340-347.

[2] S. Baier and D.J. McClements, Impact of preferential interactions on thermal stability and gelation of bovine serum albumin in aqueous sucrose solutions, J. Agric. Food Chem. 49 (2001), 2600-2608. 
[3] X. Cao, X. Yang, Y. Liu and C. Wang, Effect of sucrose on BSA denatured aggregation at high concentration studied by the iso-conversional method and the master plots method, Journal of Thermal Analysis and Calorimetry 95 (2009), 969-976.

[4] D.C. Carter and J.X. Ho, Structure of serum albumin, Adv. Protein Chem. 45 (1994), 153-203.

[5] S. Curry, H. Mandelkow, P. Brick and N. Franks, Crystal structure of human serum albumin complexed with fatty acid reveals an asymmetric distribution of binding sites, Nat. Struct. Biol. 5 (1998), 827-835.

[6] I. Diamond and A.S. Gordon, Cellular and molecular neuroscience of alcoholism, Physiol. Rev. 77 (1997), 1-20.

[7] K.A. Dill, Dominant forces in protein folding, Biochemistry 29 (1990), 7133-7155.

[8] Y. Fujita, A. Miyanaga and Y. Noda, Effect of alcohols on the thermal denaturation of Lysozyme as measured by differential scanning calorimetry, Bull. Chem. Soc. Jpn. 52 (1979), 3659-3662.

[9] J. Goral, J. Karavitis and E.J. Kovacs, Exposure-dependent effects of ethanol on the innate immune system, Alcohol 42 (2008), 237-247.

[10] J. Janatova, J.K. Fuller and M.J. Hunter, The heterogeneity of bovine albumin with respect to sulfhydryl and dimer content, J. Biol. Chem. 243 (1968), 3612-3622.

[11] N. El Kadi, N. Taulier, J.Y. Le Huerou, M. Gindre, W. Urbach, I. Nwigwe, P.C. Kahn and M. Waks, Unfolding and refolding of bovine serum albumin at acid pH: ultrasound and structural studies, Biophys. J. 91 (2006), 3397-3404.

[12] J.C. Lee and S.N. Timasheff, The stabilization of proteins by sucrose, J. Biol. Chem. 256 (1981), 7193-7201.

[13] A. Michnik, DSC study of the association of ethanol with human serum albumin, Journal of Thermal Analysis and Calorimetry 87 (2007), 91-96.

[14] M. Perham, J. Liao and P. Wittung-Stafshede, Differential effects of alcohols on conformational switchovers in alphahelical and beta-sheet protein models, Biochemistry 45 (2006), 7740-7749.

[15] P. Phelan, A.A. Gorfe, I. Jelesarov, D.N. Marti, J. Warwicker and H.R. Bosshard, Salt bridges destabilize a leucine zipper designed for maximized ion pairing between helices, Biochemistry 41 (2002), 2998-3008.

[16] R. Thirumangalathu, S. Krishnan, D.N. Brems, T.W. Randolph and J.F. Carpenter, Effects of pH, temperature, and sucrose on benzyl alcohol-induced aggregation of recombinant human granulocyte colony stimulating factor, J. Pharm. Sci. 95 (2006), 1480-1497. 


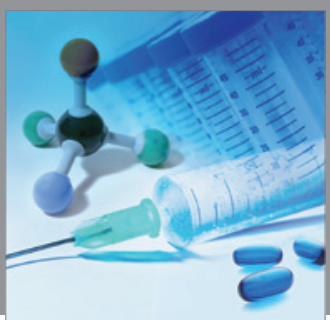

International Journal of

Medicinal Chemistry

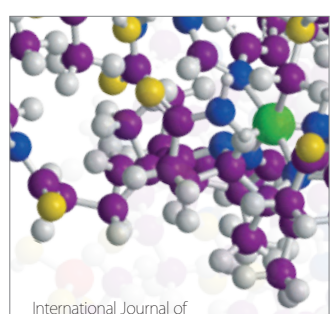

Carbohydrate Chemistry

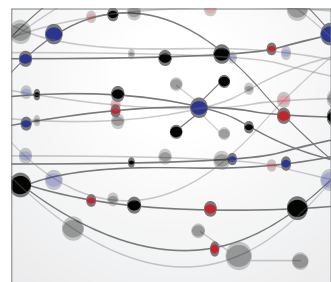

The Scientific World Journal
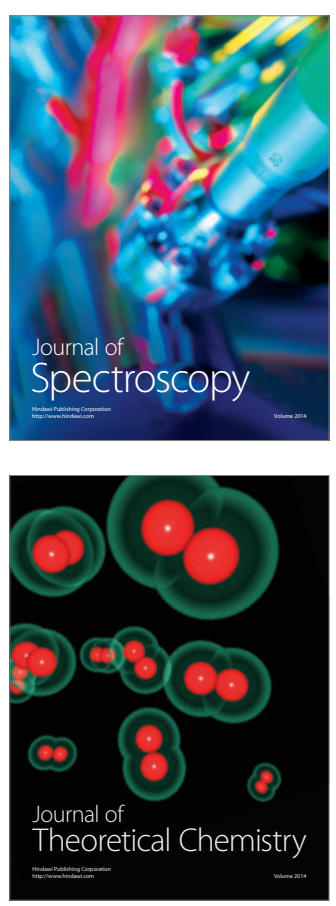
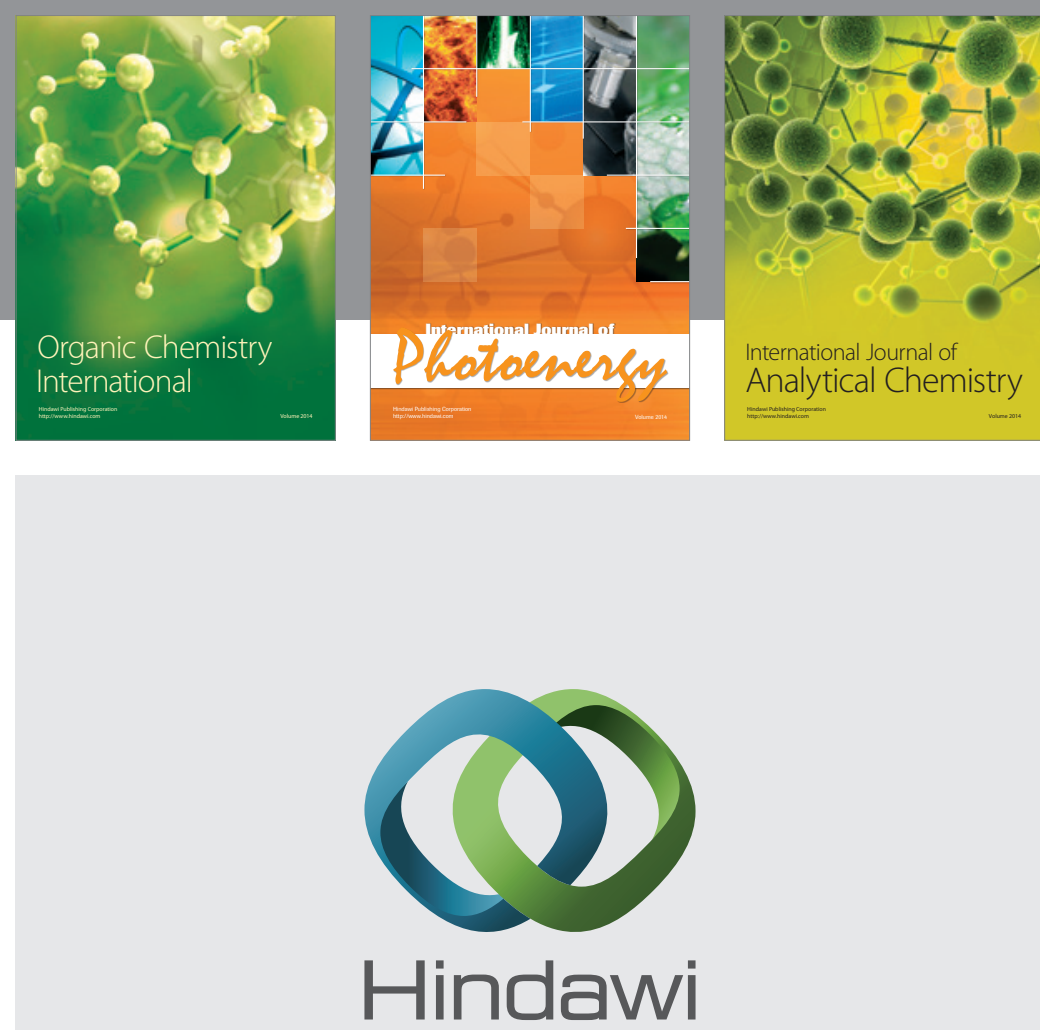

Submit your manuscripts at

http://www.hindawi.com
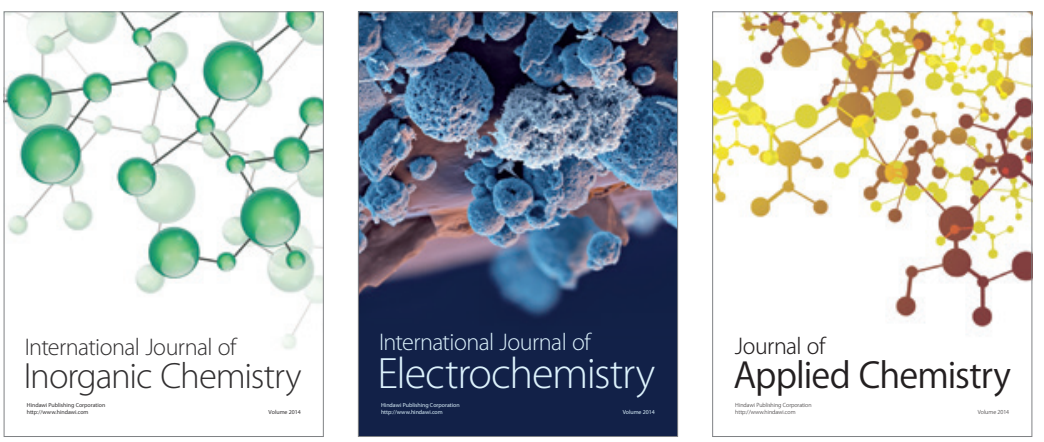

Journal of

Applied Chemistry
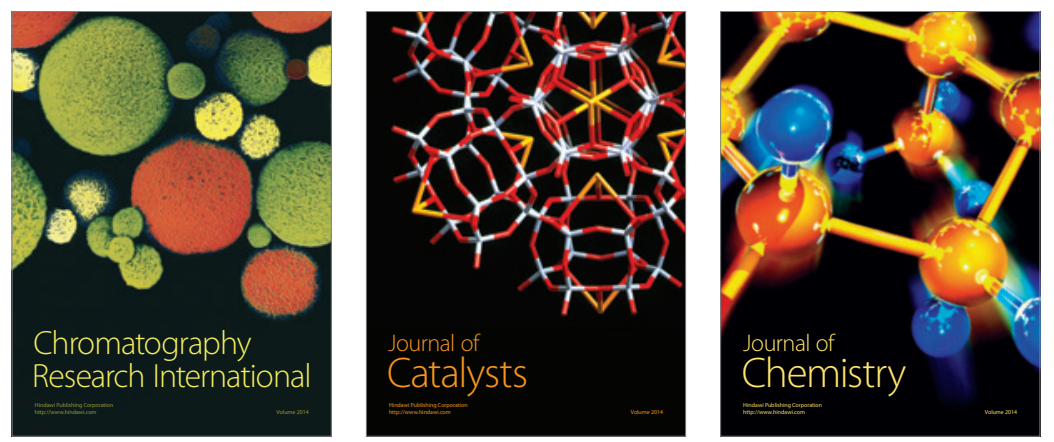
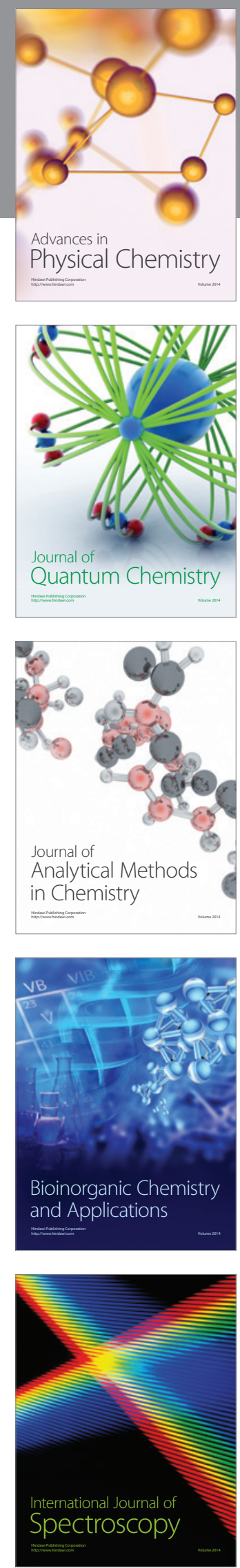